\title{
Independent prognostic miRNAs for bladder urothelial carcinoma
}

\author{
YINGBO HU, CHENG CHENG, ZHENGDONG HONG and ZIMING SHI \\ Department of Urology, The Second Affiliated Hospital of Nanchang University, \\ Nanchang, Jiangxi 330006, P.R. China
}

Received March 13, 2017; Accepted June 19, 2017

DOI: $10.3892 / 01.2017 .6471$

\begin{abstract}
Bladder cancer is the most common malignant tumor of the urinary system, and it is also an important cause of death by cancer globally. Increasing number of studies have shown that miRNAs can be used as prognostic markers for cancers. This study made use of the data available in the Cancer Genome Atlas in order to statistically analyze reported expression levels of miRNAs in samples from bladder urothelial carcinoma patients. Clinical features from a total of 399 patients and the expression data for 1,581 kinds of miRNA were included in the study. Single factor Cox regression analysis was used to identify miRNAs related to survival times for the patients. Then, through multifactors Cox regression we sorted out the independent prognostic miRNAs for the carcinoma. According to our results, 19 miRNAs were closely related to the survival times of patients with bladder urothelial carcinoma, and 3 miRNAs including hsa-mir-518b ( $\mathrm{p}=0.02)$, hsa-mir-192 $(\mathrm{p}=0.04)$ and hsa-mir-7705 ( $\mathrm{p}=0.04)$ should be useful as independent prognostic factors in patients. In addition, the survival time of those expressing high levels of hsa-mir-7705 and hsa-mir-192 was less than the survival time of those with low expression levels. However, there were no obvious differences in the survival times between high and low expressors of hsa-mir-518b. According to our results, hsa-mir-7705, hsa-mir-192 and hsa-mir-518b can be applied as independent prognostic markers for bladder urothelial carcinoma.
\end{abstract}

\section{Introduction}

Bladder cancer is a malignancy of the bladder mucosa; it is the most common malignancy of the urinary system. Its incidence ranks top among all urogenital tumors in China, and ranks second in Western countries only to prostate cancer (1-5). Bladder urothelial carcinoma is diagnosed mainly via cystoscopy $(6,7)$.

Correspondence to: Professor Ziming Shi, Department of Urology, The Second Affiliated Hospital of Nanchang University, 1 Mingde Road, Nanchang, Jiangxi 330006, P.R. China

E-mail: sen9hv@163.com

Key words: prognostic markers, miRNAs, bladder urothelial carcinoma, cancer, The Cancer Genome Atlas
miRNAs are a class of evolutionarily conserved non-coding small molecule RNAs that function by regulating gene expression at the translational level; the specific biological targets of most miRNAs remain to be discovered $(8,9)$. miRNAs have been found to regulate cell growth and tissue differentiation, and thus play roles during development and can be involved in the establishment of diseases such as cancer (10). A series of studies have shown that microRNAs play roles in cell growth and apoptosis, hemocytes differentiation, homeobox gene regulation, neuronal polarity, insulin secretion, cerebral morphogenesis, cardiac development and postembryonic development (11-14). Active research focuses on the association between miRNAs and different tumor development.

This study was designed in order to analyze the information contained in The Cancer Genome Atlas (TCGA) for bladder urothelial carcinoma patients, so as to identify independent prognostic miRNAs that should be useful as reference markers for the diagnosis of the cancer.

\section{Materials and methods}

The miRNA data for bladder urothelial carcinoma patients were downloaded from TCGA in the USA. The data involved cases from 1991 to 2013. Overall the tissue specimen data of 418 patients diagnosed with bladder urothelial carcinoma as well as the data for 19 cases of para-carcinoma tissue specimens were obtained from TCGA. To study the association between miRNA expression and clinical features in patients with bladder urothelial carcinoma in an accurate manner, we selected the patient data according to the following criteria: The age of the patient was available, the survival time was longer than 1 month and pathology had confirmed the diagnosis of bladder urothelial carcinoma. After exclusion, a total of 399 cases of bladder urothelial carcinoma and 19 cases with para-carcinoma tissue specimens were included in this study. The available clinical features included age, sex, race, the American Joint Committee on Cancer (AJCC) TNM staging system classification, the survival time and the survival status.

The miRNA expression data for the samples of 399 bladder urothelial carcinoma patients and 19 para-carcinoma specimens were included in the analysis, and a total of 1,581 miRNAs were detected in tissue specimens by using the Illumina HiSeq platform and the Illumina Genome Analyzer platform. To screen out the differentially expressed miRNAs, we set the screening criteria as log fold-change $>2$ and the associated $\mathrm{P}<0.01$ 
Table I. Clinical features of bladder urothelial carcinoma.

\begin{tabular}{|c|c|}
\hline Characteristics & Data (TCGA) \\
\hline \multicolumn{2}{|l|}{ Age } \\
\hline$\leq 65$ & 160 \\
\hline$>65$ & 239 \\
\hline \multicolumn{2}{|l|}{ Sex } \\
\hline Female & 105 \\
\hline Male & 294 \\
\hline \multicolumn{2}{|l|}{ Race } \\
\hline White & 318 \\
\hline Asian & 40 \\
\hline Black or African American & 23 \\
\hline NA & 18 \\
\hline \multicolumn{2}{|l|}{ Pathology stage } \\
\hline Stage I & 2 \\
\hline Stage II & 125 \\
\hline Stage III & 137 \\
\hline Stage IV & 133 \\
\hline NA & 2 \\
\hline \multicolumn{2}{|l|}{ T stage } \\
\hline T0 & 1 \\
\hline $\mathrm{T} 1$ & 3 \\
\hline $\mathrm{T} 2$ & 114 \\
\hline $\mathrm{T} 3$ & 191 \\
\hline $\mathrm{T} 4$ & 58 \\
\hline $\mathrm{TX}$ & 1 \\
\hline NA & 31 \\
\hline \multicolumn{2}{|l|}{$\mathrm{N}$ stage } \\
\hline No & 229 \\
\hline N1 & 46 \\
\hline $\mathrm{N} 2$ & 76 \\
\hline $\mathrm{N} 3$ & 7 \\
\hline NA & 5 \\
\hline NX & 36 \\
\hline \multicolumn{2}{|l|}{ M stage } \\
\hline M0 & 188 \\
\hline M1 & 10 \\
\hline MA & 198 \\
\hline MX & 3 \\
\hline \multicolumn{2}{|l|}{ Survival state } \\
\hline Survive & 223 \\
\hline Dead & 176 \\
\hline
\end{tabular}

TCGA, The Cancer Genome Atlas.

miRNAs that were upregulated and downregulated in the tissue specimens of bladder urothelial carcinoma patients were screened out, and the expression of miRNA was normalized.

In addition, we transformed the miRNA expression levels into $\log 2$, and screened out the miRNAs and clinical features that were associated with the survival of bladder urothelial carcinoma using the univariate Cox proportional hazards regression. Then, we used the multivariate Cox proportional hazards regression to screen out the independent prognostic
Table II. The top ten miRNA of upregulated expression.

\begin{tabular}{lcccc}
\hline miRNA & logFC & $\log C \mathrm{CP}$ & P-value & FDR \\
\hline hsa-mir-210 & 4.803108853 & 9.8642203 & $3.45 \mathrm{E}-18$ & $3.63 \mathrm{E}-16$ \\
hsa-mir-96 & 3.410662815 & 5.143223988 & $1.15 \mathrm{E}-16$ & $1.07 \mathrm{E}-14$ \\
hsa-mir-18a & 2.988898835 & 5.68100551 & $6.61 \mathrm{E}-16$ & $5.50 \mathrm{E}-14$ \\
hsa-mir-183 & 2.943927749 & 13.56270017 & $2.06 \mathrm{E}-14$ & $1.25 \mathrm{E}-12$ \\
hsa-mir-130b & 2.303867052 & 5.74901106 & $2.72 \mathrm{E}-14$ & $1.59 \mathrm{E}-12$ \\
hsa-mir-141 & 2.494450708 & 11.09284497 & $1.42 \mathrm{E}-11$ & $5.49 \mathrm{E}-10$ \\
hsa-mir-33a & 2.22270633 & 4.907692995 & $7.44 \mathrm{E}-11$ & $2.67 \mathrm{E}-09$ \\
hsa-mir-345 & 2.673695186 & 4.694451091 & $1.23 \mathrm{E}-10$ & $4.23 \mathrm{E}-09$ \\
hsa-mir-182 & 2.254097092 & 14.41365672 & $1.34 \mathrm{E}-10$ & $4.49 \mathrm{E}-09$ \\
hsa-mir-301a & 2.088008339 & 4.476181049 & $1.76 \mathrm{E}-10$ & $5.79 \mathrm{E}-09$
\end{tabular}

factors of bladder urothelial carcinoma. Furthermore, we performed a Kaplan-Meier (KM) survival curve analysis on the independent prognostic miRNAs for bladder urothelial carcinoma, divided the miRNA levels into high-expression and low-expression groups with the median as the cut-off point, and processed data using the survival package of the $\mathrm{R}$ software, version 2.14.1 ( $\mathrm{R}$ development Core Team, Vienna Austria, 2009) (15).

TargetScan was used for target prediction on the independent prognostic miRNAs for bladder urothelial carcinoma. After excluding repeated target genes, cytoscape was used performing a GO enrichment analysis on target genes $(\mathrm{p}<0.05)$ to screen out ten functions with the closest relationship to target genes. Finally, KOBAS was used for KEGG pathway enrichment analysis on the target genes to screen out ten pathways with the closest association to target genes.

\section{Results}

The data from a total of 399 patients with bladder urothelial carcinoma were included in the analysis. The data for the expression of 1,581 miRNAs and a variety of clinical features can be seen in Table I. In addition, we found the up and downregulated miRNAs in bladder urothelial carcinoma. Among them, the ten most upregulated miRNAs were hsa-mir-210, hsa-mir-96, hsa-mir-18a, hsa-mir-183, hsa-mir-130b, hsa-mir141, hsa-mir-33a, hsa-mir-345, hsa-mir-182 and hsa-mir-301a (Table II), whereas, the most downregulated ones were hsa-mir-143, hsa-mir-1298, hsa-mir-139, hsa-mir-1-2, hsa-mir195, hsa-mir-1-1, hsa-mir-133a-1, hsa-mir-30a, hsa-mir-133a-2 and hsa-mir-133b (Table III).

The univariate Cox proportional risk regression shown in Table I demonstrates the clinical features of pathology $\mathrm{N}$ stage $(\mathrm{p}=1.01 \mathrm{E}-07)$, pathology M stage $(\mathrm{p}=0.0002)$, pathology $\mathrm{T}$ stage $(\mathrm{p}=0.0005)$ and patient's age $(\mathrm{p}=0.007)$ were all closely associated with the survival length for bladder urothelial carcinoma. As for the miRNAs, related to the bladder urothelial carcinoma survival, a total of 19 miRNAs stood out, of which the three most closely related ones were hsa-mir-99a $(\mathrm{p}=0.0001)$, hsa-let-7c $(\mathrm{p}=0.0004)$ and hsa-mir-100 $(\mathrm{p}=0.0005)$.

Next, a multivariate Cox proportional risk regression showed the pathology $\mathrm{N}$ stage $(\mathrm{p}=0.00007)$, hsa-mir-518b $(\mathrm{p}=0.02)$, the patient's age $(\mathrm{p}=0.03)$, hsa-mir-192 $(\mathrm{p}=0.04)$ and hsa-mir-7705 
Table III. The top ten miRNA of downregulated expression.

\begin{tabular}{lcccc}
\hline miRNA & \multirow{2}{*}{$\operatorname{logFC}$} & $\log C \mathrm{M}$ & P-value & FDR \\
\hline hsa-mir-143 & -3.599914903 & 17.8945552 & $3.72 \mathrm{E}-46$ & $5.88 \mathrm{E}-43$ \\
hsa-mir-1298 & -4.526050745 & 1.187271104 & $1.13 \mathrm{E}-39$ & $8.92 \mathrm{E}-37$ \\
hsa-mir-139 & -2.621941214 & 6.101969894 & $9.29 \mathrm{E}-39$ & $4.89 \mathrm{E}-36$ \\
hsa-mir-1-2 & -3.736158674 & 5.508819647 & $4.22 \mathrm{E}-34$ & $1.67 \mathrm{E}-31$ \\
hsa-mir-195 & -2.432143312 & 4.989973255 & $6.44 \mathrm{E}-33$ & $2.04 \mathrm{E}-30$ \\
hsa-mir-1-1 & -3.693697839 & 5.417492946 & $1.41 \mathrm{E}-32$ & $3.73 \mathrm{E}-30$ \\
hsa-mir-133a-1 & -3.558543939 & 5.92141089 & $2.24 \mathrm{E}-28$ & $5.06 \mathrm{E}-26$ \\
hsa-mir-30a & -2.228925868 & 13.94817286 & $6.07 \mathrm{E}-27$ & $1.20 \mathrm{E}-24$ \\
hsa-mir-133a-2 & -3.515469057 & 5.736507 & $2.23 \mathrm{E}-26$ & $3.92 \mathrm{E}-24$ \\
hsa-mir-133b & -3.88838679 & 4.46304315 & $3.14 \mathrm{E}-26$ & $4.97 \mathrm{E}-24$ \\
\hline
\end{tabular}

Table IV. Univariate and multivariate analyses.

\begin{tabular}{lcccccc}
\hline & \multicolumn{2}{c}{$\begin{array}{c}\text { Univariate } \\
\text { analysis }\end{array}$} & & \multicolumn{2}{c}{$\begin{array}{c}\text { Multivariate } \\
\text { analysis }\end{array}$} \\
\cline { 2 - 3 } \cline { 5 - 6 } Variables & $\begin{array}{c}\text { HR } \\
(95 \% \mathrm{CI})\end{array}$ & P-value & & $\begin{array}{c}\text { HR } \\
(95 \% \mathrm{CI})\end{array}$ & P-value \\
\hline Pathology N stage & 0.87 & 0.0000001 & & 0.78 & 0.00007 \\
hsa-mir-518b & 0.08 & 0.04 & & 0.1 & 0.02 \\
Age & 0.49 & 0.007 & & 0.42 & 0.03 \\
hsa-mir-192 & -0.17 & 0.01 & & -0.17 & 0.04 \\
hsa-mir-7705 & -0.22 & 0.04 & & -0.24 & 0.04 \\
\hline
\end{tabular}

CI, confidence interval.

Table V. The analysis of GO enrichment.

\begin{tabular}{|c|c|c|c|}
\hline GO-ID & P-value & $\begin{array}{c}\text { Corr } \\
\text { P-value }\end{array}$ & Description \\
\hline 30528 & $1.28 \mathrm{E}-10$ & $1.86 \mathrm{E}-07$ & Transcription regulator activity \\
\hline 6357 & $4.58 \mathrm{E}-07$ & $2.29 \mathrm{E}-04$ & $\begin{array}{l}\text { Regulation of transcription from } \\
\text { RNA polymerase II promoter }\end{array}$ \\
\hline 45449 & 7.35E-07 & $2.29 \mathrm{E}-04$ & Regulation of transcription \\
\hline 16564 & 7.93E-07 & $2.29 \mathrm{E}-04$ & Transcription repressor activity \\
\hline 3676 & $8.48 \mathrm{E}-07$ & $2.29 \mathrm{E}-04$ & Nucleic acid binding \\
\hline 19219 & $9.42 \mathrm{E}-07$ & $2.29 \mathrm{E}-04$ & $\begin{array}{l}\text { Regulation of nucleobase, nucleoside, } \\
\text { nucleotide and nucleic acid } \\
\text { metabolic process }\end{array}$ \\
\hline 51171 & $1.18 \mathrm{E}-06$ & $2.45 \mathrm{E}-04$ & $\begin{array}{l}\text { Regulation of nitrogen compound } \\
\text { metabolic process }\end{array}$ \\
\hline 6355 & $1.92 \mathrm{E}-06$ & $3.49 \mathrm{E}-04$ & $\begin{array}{l}\text { Regulation of transcription, } \\
\text { DNA-dependent }\end{array}$ \\
\hline 5634 & $2.71 \mathrm{E}-06$ & 4.39E-04 & Nucleus \\
\hline 51252 & $3.24 \mathrm{E}-06$ & $4.62 \mathrm{E}-04$ & Regulation of RNA metabolic process \\
\hline
\end{tabular}

$(\mathrm{p}=0.04)$ were all independent prognostic factors for bladder urothelial carcinoma (Table IV). We performed KM survival curve analyses on the three independent prognostic miRNAs. As shown in Figs. 1-3, there were significant differences in

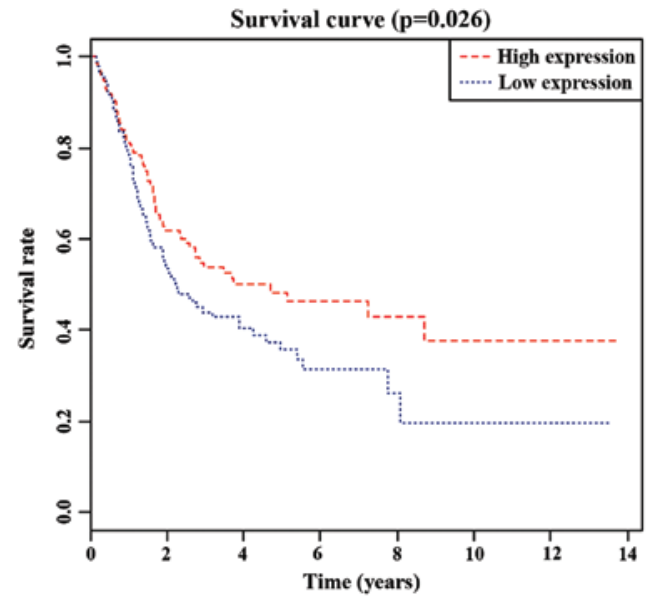

Figure 1. Survival curve of groups with highest and lowest expression levels of hsa-mir-192.

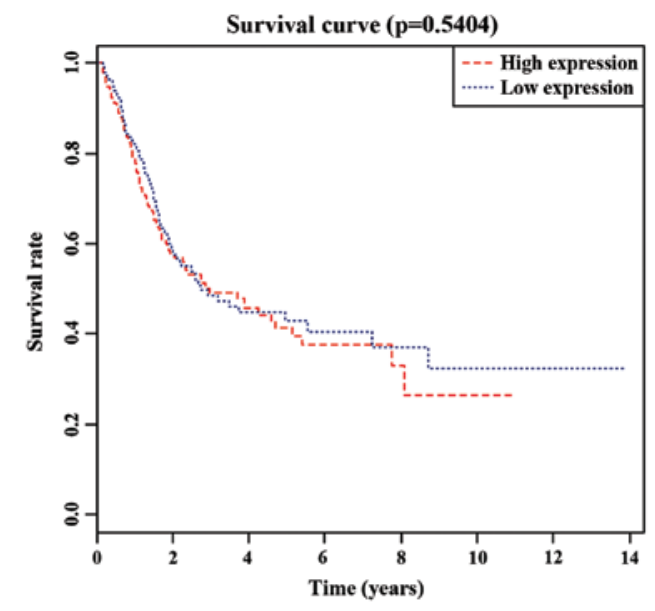

Figure 2. Survival curve of groups with highest and lowest expression levels of hsa-mir-518b.

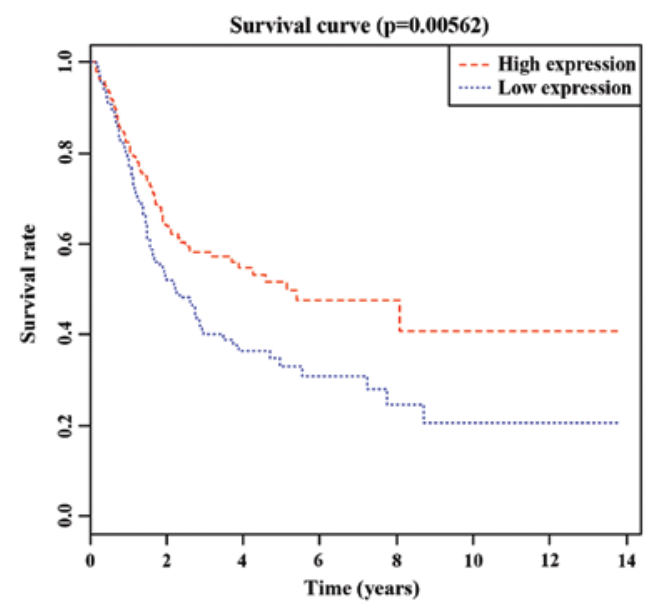

Figure 3. Survival curve of groups with highest and lowest expression levels of hsa-mir-7705.

the survival time between the high- and low-expression levels of hsa-mir-7705 and hsa-mir-192, while the survival time between the high- and low-expression levels of hsa-mir-518b did not differ significantly. 
Table VI. The analysis of KEGG enrichment.

\begin{tabular}{|c|c|c|c|c|}
\hline Term & Database & ID & P-value & $\begin{array}{l}\text { Corrected } \\
\text { P-value }\end{array}$ \\
\hline Cytokine-cytokine receptor interaction & KEGG PATHWAY & hsa04060 & 0.000784246 & 0.025901739 \\
\hline TGF- $\beta$ signaling pathway & KEGG PATHWAY & hsa04350 & 0.001564908 & 0.025901739 \\
\hline PI3K-Akt signaling pathway & KEGG PATHWAY & hsa04151 & 0.001619992 & 0.025901739 \\
\hline mRNA surveillance pathway & KEGG PATHWAY & hsa03015 & 0.001865223 & 0.025901739 \\
\hline $\mathrm{NF}-\kappa \mathrm{B}$ signaling pathway & KEGG PATHWAY & hsa04064 & 0.00190454 & 0.025901739 \\
\hline Sphingolipid signaling pathway & KEGG PATHWAY & hsa04071 & 0.003163201 & 0.035849606 \\
\hline Apoptosis & KEGG PATHWAY & hsa04210 & 0.004187233 & 0.040675974 \\
\hline Transcriptional misregulation in cancer & KEGG PATHWAY & hsa05202 & 0.00677533 & 0.049549952 \\
\hline Herpes simplex infection & KEGG PATHWAY & hsa05168 & 0.007212651 & 0.049549952 \\
\hline Chemokine signaling pathway & KEGG PATHWAY & hsa04062 & 0.007286758 & 0.049549952 \\
\hline
\end{tabular}

KEGG, Kyoto Encyclopedia of Genes and Genomes; TGF- $\beta$, transforming growth factor- $\beta$; PI3K, phosphoinositide 3-kinase; NF- $\mathrm{kB}$, nuclear factor-кB.

Finally, we used TargetScan to predict the target genes of three independent prognostic miRNAs, and then used Cytoscape to perform a GO enrichment analysis on all target genes. As shown in Table V, the three most significant functions found were transcription regulator activity, regulation of transcription from RNA polymerase II promoter and regulation of transcription. The KEGG pathway analysis revealed that the most closely related pathway was Cytokine-cytokine receptor interaction (Table VI).

\section{Discussion}

Bladder cancer is the most common malignancy of the urinary system. Studies have found that miRNAs can regulate the expression of tens to hundreds of genes, and that miRNAs play a hub role in tumorigenesis and progression as regulatory molecules in the processes of gene expression and protein translation $(16,17)$. Although these endogenous miRNAs only account for $2 \%$ of the total human transcripts, they regulate the expression of $>30 \%$ of the genes in the whole human genome. Therefore, research on the function of miRNAs and their association to the development of cancer is of great significance. This study identified independent prognostic factors of bladder urothelial carcinoma by studying the expression data of miRNAs.

We extracted the miRNA expression data of bladder urothelial carcinoma from the free and open TCGA. After screening, we listed the ten most significantly upregulated and downregulated miRNAs in the specimens, of which the most pronouncedly upregulated ones were hsa-mir-210, hsa-mir-96 and hsa-mir-18a. The level of hsa-miR-210 has been associated with hypoxia in head and neck tumors and other indicators, and a high hsa-miR-210 expression is closely related to disease recurrence and overall survival time (18). In addition, another study showed that hsa-miR-210 is an independent prognostic marker for breast cancer, and that hsa-miR-210 overexpression is induced by hypoxia in a HIF-1 $\alpha$ - and VHL-dependent manner (19). Then, the miRNA hsa-miR-96 can upregulate the levels of IRS1 and MAP4K1 to affect bladder cancer cell growth and has been signaled as a promising diagnostic marker for human bladder urothelial carcinomas (20). Interestingly, hsa-mir-18a has also been shown to be involved in many cancer processes. A study demonstrated that hsa-mir-18a was able to prevent estrogen receptor- $\alpha$ expression and promote hepatoma cell proliferation (21).

In addition, we screened out the three best independent prognostic miRNAs for bladder urothelial carcinoma by constructing a multivariate Cox regression model, and obtained hsa-mir-518b ( $\mathrm{p}=0.02)$, hsa-mir-192 ( $\mathrm{p}=0.04)$ and hsa-mir-7705 $(\mathrm{p}=0.04)$. Furthermore, by plotting the KM survival curve, we found that the survival time of patients expressing high hsa-mir-7705 and hsa-mir-192 levels were significantly shorter than those of patients expressing low levels. In addition, both hsa-mir-7705 and hsa-mir-192 were upregulated in the bladder urothelial carcinoma samples. A study in mice showed that together with elevated transforming growth factor- $\beta$ (TGF- $\beta$ ) and Colla2 levels, the levels of miR-192 are significantly higher in the glomeruli of diabetic mice when compared to the levels in non-diabetic controls, these finding suggest that miRNAs in the kidney play a role downregulating E-box repressors to control the TGF- $\beta$-induced Colla2 expression (22).

Finally, we predicted target genes for the three independent prognostic miRNAs, and then subjected those target genes to GO enrichment analysis using Cytoscape. The transcription regulator activity was the most closely associated enrichment area, while the other findings included transcription from RNA polymerase II promoter and regulation of transcription. The KEGG pathway results pointed to three associated pathways including Cytokine-cytokine receptor interactions, TGF- $\beta$, and PI3K-Akt signaling pathways. According to Kaminska et al TGF- $\beta$ signaling inhibitors may be promising novel, potential antitumor therapeutics as they decrease glioma viability and invasion in animal models (23). In addition, other researchers have shown possible initiation of pathological changes in osteoarthritis by high concentrations of active TGF- $\beta 1$ in subchondral bone, and a potential approach for managing this disease may be to suppress TGF- $\beta 1$ (24). Finally, the PI3K-Akt signaling pathway has also been studied, and it is thought that phosphoinositide 3-kinases (PI3Ks) are involved in a variety of tumor processes (25).

Even though this study identified three independent prognostic miRNAs, we are aware of the study's limitations. 
Firstly, all 399 cases of bladder urothelial carcinoma were from a single database (TCGA), so verification with data from another database is needed. On the same line, 399 cases are a rather small group size; so larger sample verification is needed. Finally, PCR validation assays will definitely enhance the reliability of our findings.

In conclusion, our approach was successful in identifying hsa-mir-7705, hsa-mir-192 and hsa-mir-518b as independent prognostic factors for bladder urothelial carcinoma.

\section{References}

1. Babjuk M, Burger M, Zigeuner R, Shariat SF, van Rhijn BW, Compérat E, Sylvester RJ, Kaasinen E, Böhle A, Palou Redorta J, et al; European Association of Urology: EAU guidelines on non-muscle-invasive urothelial carcinoma of the bladder: Update 2013. Eur Urol 64: 639-653, 2013.

2. Cancer Genome Atlas Research Network: Comprehensive molecular characterization of urothelial bladder carcinoma. Nature 507: 315-322, 2014.

3. Sternberg CN, Skoneczna I, Kerst JM, Albers P, Fossa SD, Agerbaek M, Dumez H, de Santis M, Théodore C, Leahy MG, et al; European Organisation for Research and Treatment of Cancer Genito-Urinary Cancers Group; Groupe d'Etude des Tumeurs Urogénitales; National Cancer Research Institute Bladder Cancer Study Group; National Cancer Institute of Canada Clinical Trials Group; German Association of Urologic Oncology: Immediate versus deferred chemotherapy after radical cystectomy in patients with pT3-pT4 or N+ M0 urothelial carcinoma of the bladder (EORTC 30994): An intergroup, open-label, randomised phase 3 trial. Lancet Oncol 16: 76-86, 2015.

4. Sanford T, Porten S and Meng MV: Molecular analysis of upper tract and bladder urothelial carcinoma: Results from a microarray comparison. PLoS One 10: e0137141, 2015.

5. Liu J, Cai M, Chen J, Liao Y, Mai S, Li Y, Huang X, Liu Y, Zhang J, Kung H, et al: $\alpha 4$ contributes to bladder urothelial carcinoma cell invasion and/or metastasis via regulation of E-cadherin and is a predictor of outcome in bladder urothelial carcinoma patients. Eur J Cancer 50: 840-851, 2014.

6. Sahin S, Resorlu B, Atar FA, Eksi M, Sener NC and Tugcu V: Laparoscopic ureterolithotomy with concomitant pyelolithotomy using flexible cystoscope. Urol J 13: 2833-2836, 2016.

7. Mahmood N and Pasha T: A new technique to prevent curling of guide wire in urinary bladder during $\mathbf{J}$ stent insertion with flexible cystoscope. Can Urol Assoc J 10: E34-E35, 2016.

8. Frampton GM, Fichtenholtz A, Otto GA, Wang K, Downing SR, He J, Schnall-Levin M, White J, Sanford EM, An P, et al: Development and validation of a clinical cancer genomic profiling test based on massively parallel DNA sequencing. Nat Biotechnol 31: 1023-1031, 2013.

9. Köttgen A, Albrecht E, Teumer A, Vitart V, Krumsiek J, Hundertmark C, Pistis G, Ruggiero D, O'Seaghdha CM, Haller T, et al; LifeLines Cohort Study; CARDIoGRAM Consortium; DIAGRAM Consortium; ICBP Consortium; MAGIC Consortium: Genome-wide association analyses identify 18 new loci associated with serum urate concentrations Nat Genet 45: 145-154, 2013.
10. Nunez-Iglesias J, Liu CC, Morgan TE, Finch CE and Zhou XJ: Joint genome-wide profiling of miRNA and mRNA expression in Alzheimer's disease cortex reveals altered miRNA regulation. PLoS One 5: e8898, 2010.

11. Khorshid M, Hausser J, Zavolan M and van Nimwegen E: A biophysical miRNA-mRNA interaction model infers canonical and noncanonical targets. Nat Methods 10: 253-255, 2013.

12. Muniategui A, Pey J, Planes FJ and Rubio A: Joint analysis of miRNA and mRNA expression data. Brief Bioinform 14: 263-278, 2013

13. Luo D, Wilson JM, Harvel N, Liu J, Pei L, Huang S, Hawthorn L and Shi H: A systematic evaluation of miRNA: mRNA interactions involved in the migration and invasion of breast cancer cells. J Transl Med 11: 57, 2013.

14. Gu P, Reid JG, Gao X, Shaw CA, Creighton C, Tran PL, Zhou X, Drabek RB, Steffen DL, Hoang DM, et al: Novel microRNA candidates and miRNA-mRNA pairs in embryonic stem (ES) cells. PLoS One 3: e2548, 2008.

15. Baayen RH: Analyzing Linguistic Data: A Practical Introduction to Statistics Using R. Cambridge University Press, New York, 2008. https://doi.org/10.1017/CBO9780511801686.

16. Liu B, Li J and Tsykin A: Discovery of functional miRNA-mRNA regulatory modules with computational methods. J Biomed Inform 42: 685-691, 2009.

17. Tonevitsky AG, Maltseva DV, Abbasi A, Samatov TR, Sakharov DA, Shkurnikov MU, Lebedev AE, Galatenko VV, Grigoriev AI and Northoff $\mathrm{H}$ : Dynamically regulated miRNA-mRNA networks revealed by exercise. BMC Physiol 13: 9, 2013.

18. Gee HE, Camps C, Buffa FM, Patiar S, Winter SC, Betts G, Homer J, Corbridge R, Cox G, West CM, et al: hsa-mir-210 is a marker of tumor hypoxia and a prognostic factor in head and neck cancer. Cancer 116: 2148-2158, 2010.

19. Camps C, Buffa FM, Colella S, Moore J, Sotiriou C, Sheldon H, Harris AL, Gleadle JM and Ragoussis J: hsa-miR-210 is induced by hypoxia and is an independent prognostic factor in breast cancer. Clin Cancer Res 14: 1340-1348, 2008.

20. Wang Y, Luo H, Li Y, Chen T, Wu S and Yang L: hsa-miR-96 up-regulates MAP4K1 and IRS1 and may function as a promising diagnostic marker in human bladder urothelial carcinomas. Mol Med Rep 5: 260-265, 2012

21. Liu WH, Yeh SH, Lu CC, Yu SL, Chen HY, Lin CY, Chen DS and Chen PJ: MicroRNA-18a prevents estrogen receptor- $\alpha$ expression, promoting proliferation of hepatocellular carcinoma cells. Gastroenterology 136: 683-693, 2009.

22. Kato M, Zhang J, Wang M, Lanting L, Yuan H, Rossi JJ and Natarajan R: MicroRNA-192 in diabetic kidney glomeruli and its function in TGF- $\beta$-induced collagen expression via inhibition of E-box repressors. Proc Natl Acad Sci USA 104: 3432-3437, 2007.

23. Kaminska B, Kocyk M and Kijewska M: TGF beta signaling and its role in glioma pathogenesis. Adv Exp Med Biol 986: 171-187, 2013.

24. Zhen G, Wen C, Jia X, Li Y, Crane JL, Mears SC, Askin FB, Frassica FJ, Chang W, Yao J, et al: Inhibition of TGF- $\beta$ signaling in mesenchymal stem cells of subchondral bone attenuates osteoarthritis. Nat Med 19: 704-712, 2013.

25. Martini M, De Santis MC, Braccini L, Gulluni F and Hirsch E: PI3K/AKT signaling pathway and cancer: An updated review. Ann Med 46: 372-383, 2014. 